\title{
Percepción por los estudiantes de la divulgación científica en la prensa y de su contribución a la cultura científica
}

\author{
Begoña Cantabrana, Brezo Diez, Agustín Hidalgo
}

Introducción. Este trabajo presenta y evalúa un ejercicio formativo en relación al papel potencial de la prensa en la divulgación científica y en la apropiación de la ciencia por los ciudadanos.

Sujetos y métodos. Participaron 161 alumnos distribuidos en grupos de 5-6 para realizar el trabajo propuesto en forma de seminario. Los alumnos seleccionaron artículos de ciencia y medicina publicados en diarios nacionales o regionales para identificar en ellos términos científicos y médicos y formar opinión para manifestar su acuerdo con la siguiente afirmación: 'la debilidad de la cultura científica española se debe a su escasa divulgación'.

Resultados. Los alumnos seleccionaron 103 artículos e identificaron 294 términos, la mayoría relacionados con enfermedades y sólo seis con ciencias naturales. Todos los grupos coincidieron en la baja cultura científica de los españoles; el 61,29\% la atribuyó a la escasa divulgación científica, mientras que el 38,71\% restante consideró que la divulgación es adecuada, hay información suficiente en los diarios y en un número apreciable de revistas, además de televisión e Internet.

Conclusión. Entre los alumnos existe una opinión unánime en que la cultura científica es reducida, pero no en las causas. Sin embargo, la mayoría coincide en que puede deberse a unos planes de estudios que no promueven adecuadamente la ciencia ni la cultura científica, además del poco interés de la población y de las autoridades gubernamentales en su promoción y difusión. El interés y la motivación personal pueden explicar la divergencia de opinión entre los alumnos.

Palabras clave. Cultura científica. Divulgación científica. Estudiantes de medicina. Terminología científica.

Students' perception of the scientific diffusion in the press and of its contribution to the scientific culture

Departamento de Medicina. Facultad de Medicina y Ciencias de la Salud. Universidad de Oviedo. Oviedo, Asturias, España.

Correspondencia:

Dra. Begoña Cantabrana. Farmacología. Departamento de Medicina. Facultad de Medicina. Universidad de Oviedo. Julián Clavería, 6. E-33006 Oviedo (Asturias).

Fax: +34985103551.

E-mail:

bego@uniovi.es

Conflicto de intereses: No declarado.

Competing interests: None declared.

(c) 2015 FEM

Introduction. This study presents and evaluates a formative exercise about the potential role of the press in scientific diffusion and in the social appropriation of science.

Subjects and methods. 161 students participated in the study distributed in groups of five or six to perform the proposed assignments in a seminar form. Medicine and science articles published in national and regional newspapers were selected by the students to identify specific medical and scientific terms and to have their own opinion to agree with the statement: 'the weakness of Spanish scientific culture is due to its limited diffusion'.

Results. 103 articles were selected and 294 terms identified, most of them related with diseases and only six with natural sciences. All groups agree with the low scientific culture of the Spanish people. A 61.29\% attributed this to scarce scientific communication, whereas the other $38.71 \%$ considered that scientific diffusion is adequate due to there is enough information in newspapers and magazines besides television and Internet.

Conclusion. There was no unanimity about the causes of scientific backwardness. However, most students agree that it could be attributed to the little interest of Government authorities and other people in promoting science and scientific culture, what in the end is reflected also in curricula. In this study, the interest and personal motivation of each student may explain the different views between them.

Key words. Diffusion of science. Scientific culture. Scientific terminology. Students of medicine.

\section{Introducción}

En los últimos años, diversos autores han contribuido a dilucidar algunos aspectos de la aportación de España a la producción de conocimiento y a ana- lizar el tantas veces proclamado atraso de la ciencia y la cultura científica española. Mediante estos estudios, se ha establecido que la producción científica española fue poco relevante a partir de que la primera revolución científica no tuviera en España 
la misma repercusión que en otros países debido a cuestiones políticas, económicas y sociales mediatizadas por las políticas particulares de los sucesivos gobernantes [1]. Este hecho motivó tanto la emigración de científicos como la dependencia científica y tecnológica del exterior [2,3] en determinados períodos históricos. Afortunadamente, las políticas científicas desarrolladas a partir de los años ochenta han consolidado infraestructuras de investigación y mejorado la producción científica [4-6]. Además, si bien cada época ha utilizado medios de divulgación científica, entendida ésta como cualquier proceso de transmisión del conocimiento, en España, estos procesos han sido poco eficientes y generalmente han seguido el modelo de 'déficit cognitivo' mediante una divulgación unidireccional con escasa participación popular hasta la primera mitad del siglo xx [7,8].

Por otra parte, se ha ido consolidando la idea de que la ciencia es parte medular de la cultura puesto que 'el papel cultural de la ciencia podría rescatar a la cultura de su tendencia a la banalización' [9]. En el mismo sentido, se ha entendido la ciencia como un elemento cultural porque 'ya no van quedando cosas importantes que hacer que no dependan de la ciencia y la tecnología' [10]. Por eso, la alfabetización científica es cada día más importante.

La divulgación científica pretende acercar el conocimiento a otros estamentos culturales de la sociedad con, al menos, una doble finalidad: aumentar el nivel de formación de los ciudadanos y fomentar la implicación de la sociedad en el progreso científico facilitando el desarrollo de algunas investigaciones, dado que 'la legitimación de determinadas teorías requiere el beneplácito de los medios y de la opinión pública en general' [7]. De hecho, de la interacción de la ciencia y la tecnología con la sociedad emerge la cultura tecnocientífica que caracteriza el desarrollo humano actual [11, 12], en la que todos los protagonistas han de asumir nuevos papeles complementarios de los tradicionales. El reto, por tanto, de la divulgación científica a través de los medios de comunicación es la transmisión de conocimientos especializados, que se producen dentro de una comunidad de expertos, a un público más amplio a fin de aumentar su bagaje cultural.

El conocimiento científico ha venido a convertirse en un factor determinante tanto de la actitud del ciudadano ante la ciencia como de la conciencia social [13], aunque exista la percepción de que los grandes esfuerzos de divulgación científica en la segunda mitad del siglo xx y en este inicio de siglo no han dado los frutos deseados en la elevación de la cultura científica en la ciudadanía $[7,8,14,15]$. No obstante, sí se ha iniciado un interesante proceso de democratización de la ciencia [16] con una no despreciable apropiación social de ésta [17] que ha propiciado, al menos en el ámbito de la salud, la participación de colectivos ciudadanos en la orientación de las políticas científicas [14,18-22]. Para mantener la presencia ciudadana en la gestión de la vida pública es necesario que los ciudadanos adquieran una comprensión suficiente del entorno científico y tecnológico que les permita valorar, apoyar, sugerir y votar sobre acciones políticas encaminadas a un mejor gobierno de nuestras sociedades y a la satisfacción de las necesidades humanas [22].

Por último, investigaciones sobre épocas más recientes, centradas en el siglo $\mathrm{xx}$, han demostrado el modo en que los medios de comunicación de masas contemporáneos han desempeñado una función relevante en la elaboración de conocimiento científico, más allá de la simple difusión de los últimos descubrimientos [8]. Así mismo, se ha constatado un interés creciente por los estudios de investigación sobre la divulgación de la ciencia en España, dentro de los cuales ocupan un lugar destacado los relacionados con la medicina [23]. Sin embargo, no es conocida la percepción de este problema por los estudiantes universitarios.

Con el fin de evaluar la opinión de los alumnos sobre la cultura científica en España hemos diseñado una actividad en la que los alumnos del primer curso del grado en medicina deben identificar noticias científicas en la prensa diaria y estimar el grado de acuerdo con la afirmación 'la debilidad de la cultura científica española se debe a su escasa divulgación', una vez que han realizado un análisis de la información científica y médica contenida en la prensa nacional y regional.

\section{Sujetos y métodos}

El ejercicio se desarrolló durante el curso 20102011 en la asignatura 'Introducción a la medicina, documentación y método científico', de seis créditos ECTS, con 60 horas presenciales (58 horas de actividades presenciales y 2 horas de examen escrito) y 90 horas no presenciales, que se imparte en el primer semestre del primer curso del grado en medicina en la Universidad de Oviedo. Las actividades presenciales se distribuyeron en 28 clases teóricas (incluyendo dos conferencias invitadas), 6 horas de prácticas, 20 horas de seminarios y 2 horas de tutoría grupal. La actividad descrita en este trabajo se incluyó dentro de los seminarios. 


\section{Grupos de alumnos y programación de la tarea}

En el curso se matricularon 161 alumnos, de los que 152 eran de nueva matrícula y nueve, adaptados de la licenciatura en medicina. De ellos, 139 accedieron desde las pruebas de acceso a la universidad, ocho eran titulados universitarios, dos eran mayores de 25 años, otros dos accedieron por convalidación parcial de estudios extranjeros y uno por traslado desde otra universidad. Los 161 alumnos matriculados se distribuyeron en 32 subgrupos de cinco o seis alumnos que se mantuvieron estables a lo largo del semestre, de la misma forma que describimos en un trabajo previo [24]. En cada seminario participaron ocho subgrupos (unos 40 alumnos), un alumno de cada subgrupo debió exponer el trabajo realizado de forma conjunta y participativa. Al final de la actividad, el alumno ponente debía incorporar la presentación al portafolio del grupo. Esta información y las notas personales del profesor en el curso del seminario (exposición y debate) han sido los documentos utilizados para la realización del presente trabajo. El seminario que nos ocupa, denominado 'Identificación de términos científicos y médicos en prensa diaria, se celebró entre los días 27 de septiembre y 7 de octubre de 2011. Dentro del mismo, los alumnos debieron manifestar su acuerdo/desacuerdo con la expresión: 'la debilidad de la cultura científica española se debe a su escasa divulgación.

Al comienzo del curso los alumnos tenían disponibles en el campus virtual de la universidad las instrucciones para la realización del trabajo. Estas instrucciones contenían un objetivo (tomar conciencia de la dimensión social de la ciencia y la medicina a través de su presencia en los medios de comunicación) y una pormenorización de las tareas (analizar un periódico editado en España durante un período de al menos siete días):

- Identificar noticias relacionadas con ciencia o con medicina que aparezcan en un diario nacional o regional y extraer los términos científicos y médicos utilizados.

- A partir de la información extraída, formar opinión de grupo para manifestar su acuerdo o desacuerdo con la expresión 'la debilidad de la cultura científica española se debe a su escasa divulgación', y aportar las razones que pueden justificar la opinión manifestada.

De forma orientativa se les incluía una selección de tres noticias tomadas de la edición nacional de un diario español de gran tirada.

A efectos de cómputo hemos considerado como noticias científicas no sólo las relacionadas con las ciencias naturales (física, química, biología), sino también las referidas a cualquiera de los procesos relacionados con la salud (p. ej., promoción de la salud y autocuidados, intervenciones preventivas, aspectos culturales, sociales, políticos y económicos relacionados con la salud) y la enfermedad (p. ej., agentes causales, entidades nosológicas, manifestaciones clínicas, diagnóstico y pruebas diagnósticas, terapéutica, pronóstico, investigación clínica, traslacional e investigación básica de orientación biomédica, etc.), dado que la medicina tiene paulatinamente una base científica más amplia (sus actuaciones se basan cada vez más en la investigación y su conocimiento resultante), que acompaña a sus otras facetas como técnica (diseño, instrumentación), profesión (terapéutica) y arte (adecuada combinación de los recursos para obtener la curación del paciente o al menos el alivio de sus síntomas) [25], y así es considerado por el $89 \%$ de los ciudadanos de la Unión Europea y por el 86\% de los españoles [26].

De la misma forma hemos procedido para clasificar los términos identificados en las noticias seleccionadas. Así, por ejemplo, los que denominan 'medicamentos' los hemos considerado términos médicos terapéuticos (aunque evidentemente son entidades químicas) atendiendo a que aparecen bajo la denominación de principios activos poseedores de actividad farmacológica susceptibles de usarse en terapéutica y prescribirse con esta denominación.

El ejercicio de un subgrupo de alumnos no se incluyó en el estudio porque el trabajo no se incorporó al campus virtual y, en consecuencia, no estuvo disponible para el análisis.

\section{Resultados}

Los estudiantes identificaron y presentaron en el seminario un total de 103 artículos de los cuales, para el estudio, hemos utilizado 83 que reúnen las características de tener como objetivo claro comunicar resultados nítidos de forma divulgativa, citar las fuentes primarias donde están contenidos los datos que sustentan el artículo y que no sean publicitarios ni planteen conflictos de interés. Los artículos no incluidos se referían a noticias de congresos, notas de agencia, comunicados de empresas, entrevistas, publicidad, artículos de opinión y comentarios editoriales. Los artículos procedían de diarios nacionales y regionales y, en ellos, los alumnos identificaron 294 términos científico-médicos que pueden agruparse en diferentes ítems (Tabla I), de los que el más frecuente es el que engloba enfermedades y patologías $(20,4 \%)$; ello puede relacionarse 
Tabla I. Distribución temática de los términos científicos identificados $(n=294)$.

\begin{tabular}{lcc}
\hline & $n$ & $\%$ \\
\hline Enfermedad/patología & 60 & 20,40 \\
\hline Estructuras anatómicas macro y microscópicas & 44 & 14,96 \\
\hline Medicamentos & 41 & 13,94 \\
\hline Función y estado del paciente & 36 & 12,24 \\
\hline Aspectos generales a & 30 & 10,20 \\
\hline Sistema Nacional de Salud & 24 & 8,16 \\
\hline Instrumentos y técnicas & 22 & 7,48 \\
\hline Acto médico & 14 & 4,76 \\
\hline Especialidades médicas & 11 & 3,74 \\
\hline Terapias no farmacológicas & 6 & 2,04 \\
\hline Términos de ciencias naturales & 6 & 2,04 \\
\hline
\end{tabular}

a Vida, muerte, médico, hombre, mujer, etc.

con la elevada prevalencia de los problemas sobre salud en la sociedad actual. La relevancia cuantitativa de las noticias relacionadas con estructuras anatómicas macro y microscópicas se debe a la presencia de noticias relacionadas con el deporte y las lesiones deportivas, y con el desarrollo de la biología celular implicada en prácticamente todos los aspectos de la medicina y las ciencias biológicas. También hay una importante presencia de alusiones a medicamentos, reconocidos como un elemento terapéutico de primer nivel en comparación con las terapias no farmacológicas, cuya presencia es testimonial. Sorprende que sólo seis términos relacionados con ciencias naturales (en este caso relacionados con la física) fueran identificados por los estudiantes.

A raíz de las explicaciones aportadas, podemos afirmar que todos los grupos de estudiantes están de acuerdo en que el atraso científico de nuestra sociedad es innegable. Ningún grupo lo negó ni lo puso en duda. En lo que disienten parcialmente es en las razones de que ocurra.

Los resultados del estudio indican que el 61,3\% de los grupos de estudiantes están de acuerdo con la afirmación y admiten que la culpa de este atraso científico se debe a una escasa divulgación porque pocos medios tienen una sección fija de ciencia o de salud y cuesta encontrar este tipo de noticias. Esta escasa presencia en los medios puede explicarse, siempre según los estudiantes, por distintas razones (Tabla II):

- Falta de interés de los lectores y de los editores, que podría apoyarse en la falta de tradición histórica, el complejo lenguaje de la ciencia o la falta de interés social.

- Debilidad de los planes de estudios, dado que se dedica poco tiempo a la promoción del interés por la ciencia.

- Falta de inversión, lo que puede sugerir que no es prioritario para el Estado.

Por el contrario, el 38,7\% de los grupos de estudiantes está en desacuerdo con la afirmación porque, en su opinión, la divulgación es adecuada tanto a través de la prensa general como especializada, todos los días se producen noticias sobre ciencia y tanto la televisión (a través de programas divulgativos) como Internet son fuentes adecuadas para documentarse. En consecuencia, la divulgación no sería culpable ni habría creado el problema. Curiosamente, las razones que esgrimen estos alumnos para exculpar a la divulgación y explicar la debilidad de la cultura científica son similares a los utilizados por los alumnos que culpaban a la divulgación científica: responsabilizan a la educación, a la falta de interés y valoración de los ciudadanos y a la reducida financiación que recibe la actividad investigadora (Tabla II).

\section{Discusión}

De los alumnos participantes, el $86,3 \%(n=139)$ son de nuevo ingreso y primera matrícula en la universidad, alumnos en el rango de 17-18 años que acaban de terminar el bachillerato y superar las pruebas de acceso a la universidad con una nota de corte de 12,293 puntos sobre 14. Constituyen, por tanto, un colectivo adecuado para obtener la información exigida por el ejercicio y con garantías de capacidad intelectual y de trabajo dado el nivel de las calificaciones con que han accedido a los estudios universitarios.

A pesar de que el período de observación ha sido muy limitado, el número de artículos recogidos por los estudiantes es estimable y sugiere que todos los días puede encontrarse algún artículo que alude a aspectos científico-médicos. También es importante la amplia representación del vocabulario propio de las ciencias médicas, por lo que los medios de 
Tabla II. Distribución de los fundamentos y las explicaciones de los alumnos que manifiestan acuerdo o desacuerdo con la expresión: 'la debilidad de la cultura científica se debe a su escasa divulgación' ( $n=31$ grupos).

\begin{tabular}{|c|c|c|c|}
\hline \multicolumn{2}{|c|}{ Acuerdo $(n=19)$} & \multicolumn{2}{|c|}{ Desacuerdo $(n=12)$} \\
\hline Razones del acuerdo & Explicaciones aportadas & Razones del desacuerdo & Explicaciones aportadas \\
\hline \multirow{7}{*}{$\begin{array}{l}\text { Cuesta encontrar noticias en los medios } \\
\text { de comunicación y casi nunca en portada } \\
\text { La presencia en los medios es casi marginal } \\
\text { Poca presencia en los informativos de televisión } \\
\text { La prensa no percibe que debe divulgar la ciencia } \\
\text { Pocos medios tienen una sección de ciencia } \\
\text { No hay una cultura científica arraigada } \\
\text { que alimente la divulgación }\end{array}$} & $\begin{array}{l}\text { La terminología es muy especifica, } \\
\text { compleja y poco atractiva }\end{array}$ & \multirow{7}{*}{$\begin{array}{l}\text { La divulgación es adecuada porque: } \\
\text { - Se encuentran noticias sobre } \\
\text { ciencia con frecuencia y no hay pocas } \\
\text { - Todos los días hay noticias científicas } \\
\text { - Hay revistas especializadas accesibles } \\
\text { - Hay suficientes noticias } \\
\text { y medios especializados } \\
\text { nacionales e internacionales } \\
\text { - Todo está en Internet }\end{array}$} & \multirow{7}{*}{$\begin{array}{l}\text { La divulgación no es culpable porque: } \\
\text { - Las noticias sobre ciencia no } \\
\text { interesan a la gente: infravalorada } \\
\text { por la opinión pública } \\
\text { - Falta de financiación pública } \\
\text { y privada de la ciencia } \\
\text { - Se le concede poca importancia } \\
\text { en la educación. Debe reorientarse }\end{array}$} \\
\hline & $\begin{array}{l}\text { Falta de formación básica en los } \\
\text { colegios que no promueve la ciencia }\end{array}$ & & \\
\hline & El sistema educativo no despierta interés & & \\
\hline & Pocos periodistas especializados & & \\
\hline & $\begin{array}{l}\text { Es una responsabilidad compartida } \\
\text { entre los medios y la sociedad }\end{array}$ & & \\
\hline & $\begin{array}{l}\text { La ciencia sufre cierta estigmatización: } \\
\text { no está socialmente aceptada }\end{array}$ & & \\
\hline & Poca tradición histórica & & \\
\hline
\end{tabular}

comunicación de masas pueden considerarse un vehículo adecuado de divulgación de la terminología médica y un medio para la adquisición de vocabulario por los alumnos $[27,28]$. Asimismo, pueden contribuir a facilitar al ciudadano una apropiación social de conceptos y términos médicos $[17,29]$. La elevada presencia de términos relacionados con medicamentos ya ha sido detectada en otros estudios, como el que mantiene activo la Fundación Quiral desde hace años a través del Observatorio de Comunicación Científica de la Universitat Pompeu Fabra. En cambio, los términos propios de ciencias naturales (sobre todo de física) tienen una pobre representación en la prensa, posiblemente por su complejidad o porque se considere más adecuada para publicaciones especializadas.

La información científica y médica recogida en los medios puede servir para varios propósitos entre los que, según Díaz [21] se encuentran:

- Como vehículo de controversia científica.

- Como soporte para la formación de la imagen social de la ciencia y de la medicina.

- Como instrumento para la apropiación social de la ciencia.

- Como soporte mediático en la utilización científica y cultural de los descubrimientos.

En consecuencia, la prensa es un referente imprescindible del imaginario colectivo y marco de las con- troversias éticas que genera el desarrollo científico y tecnológico, así como una necesidad económica y cultural en la divulgación científica [30,31]. Por tanto, podemos considerar que es un instrumento adecuado para que los alumnos puedan pronunciarse sobre el papel de la divulgación en la cultura científica de la población.

Todos los grupos de estudiantes aceptan que la cultura científica en España es escasa. Esta respuesta tan contundente resulta sorprendente teniendo en cuenta que diferentes estudios han detectado un interés importante de los españoles por la ciencia $[32,33]$, en línea con el interés detectado por un estudio específico del Eurobarómetro (2007) dedicado a la investigación en los medios de comunicación. Posiblemente esta opinión puede transmitir un ambiente poco esperanzador condicionado por sus estudios precedentes, una traslación de las inquietantes circunstancias sociales asociadas a la economía o una opinión derrotista y no actualizada pero que ha calado en la población y de la que, como también han manifestado los alumnos, el tipo de educación y el escaso apoyo a la ciencia en los presupuestos generales del Estado serían responsables.

Mayoritariamente (61,3\%), los alumnos son de la opinión de que la escasa divulgación es la responsable de la baja cultura científica de la población. Fundamentan su opinión en el 'reducido' número de artículos identificados, la ausencia de una cultura que 
alimente la divulgación científica, el poco importante papel de la televisión y la ausencia de secciones fijas de ciencia en los diarios. Las explicaciones que aportan pueden agruparse en varios apartados temáticos:

- Relacionadas con las noticias: la complejidad de los textos y el lenguaje árido de algunas ciencias para los no iniciados.

- Relacionadas con la sociedad: la falta de interés, una cierta estigmatización de la ciencia, un cambio cultural con retroceso de la cultura del esfuerzo, o la falta de asociación del bienestar social a los progresos científicos.

- Educativos: la poca tradición de cultura científica, la falta de formación en ciencias básicas en la enseñanza preuniversitaria, los sistemas educativos de espalda a la ciencia y la falta de apuesta por la ciencia en los planes de estudios de todos los niveles educativos.

- Profesionales: la escasez tanto de periodistas especializados como de científicos divulgadores y una dudosa rentabilidad editorial de la divulgación científica.

Algunos de estos aspectos han sido señalados por autores como Domínguez [34], quien afirma que 'desde la prensa española se realiza muy poca divulgación científica de calidad', que los periodistas 'no sienten su responsabilidad en el momento de educar, de formar, científicamente al público' y que 'muy pocos científicos se consideran obligados a transmitir sus resultados a la sociedad. De la misma forma, Calvo [30] sostiene que 'ni los políticos, ni la generalidad de los docentes ni de los propietarios de los medios informativos manifiestan la sensibilidad de considerar la divulgación de la ciencia y la tecnología como un reto de nuestro tiempo', mientras que 'la divulgación científica debe ser considerada como un asunto de interés nacional.' También se ha llamado la atención sobre el hecho de que es necesaria una alfabetización audiovisual y multimedia para incorporar los medios al currículo escolar. Tal vez esto pueda acortar la distancia entre la formación curricular y la vocación científica [29,35-37].

Contra esta opinión negativa, el $38,71 \%$ de los estudiantes considera que la divulgación es adecuada porque es frecuente encontrar noticias sobre ciencia y medicina en los diarios, y porque hay un número apreciable de revistas científicas de divulgación y especializadas de acceso libre o mediante suscripción (hasta 17 pueden identificarse en diferentes repertorios, y alguna, como Muy Interesante, tiene una tirada de dos millones de ejemplares). Estos estudiantes, a pesar de que exculpan a la divul- gación científica, comparten con el grupo anterior las opiniones de que el sistema educativo debe reorientarse para acoger mayor formación científica, que la opinión pública infravalora el papel de la ciencia y que debe implementarse su financiación pública y privada, lo que supone un núcleo de opinión compartida. Un estudio reciente de Semir [33] ha puesto de manifiesto una serie de aspectos esperanzadores para el papel de la prensa en la divulgación científica: las búsquedas de información científica en Internet se incrementa de forma exponencial, aumenta el número de científicos que divulgan sus investigaciones en medios de comunicación de masas y la calidad de los artículos de divulgación científica escritos por periodistas especializados han sido considerados de buena calidad por los autores de los artículos originales.

En conclusión, hay dos acuerdos entre las opiniones de los estudiantes: la cultura científica española es reducida, y unos sistemas educativos inadecuados para el fomento de la ciencia, la deficiente financiación y la infravaloración de las aportaciones científicas pueden contribuir a esta inadecuada cultura científica. Por otra parte, el interés y la motivación personal pueden explicar la divergencia en la opinión de los alumnos sobre el hecho de que la divulgación sea la responsable del estado de nuestra cultura científica.

\section{Bibliografía}

1. Sánchez-Ron JM. Institucionalización de la investigación en España en el primer tercio del siglo xx: perspectiva comparada en el contexto del surgimiento de las grandes instituciones de investigación europeas. In Romero A, Santesmases MJ, eds. Cien años de política científica en España. Bilbao: Fundación BBVA; 2008. p. 23-8.

2. Gómez A, Carenas F. La vuelta de los cerebros. Barcelona: Plaza y Janés; 1976.

3. Senent-Josa J. Miseria y dependencia científica. Barcelona: Laia; 1977.

4. Sebastián J, Muñoz E, eds. Radiografía de la investigación pública en España. Madrid: Biblioteca Nueva; 2006.

5. Quintanilla MA. La política científica en la España democrática. Balance y perspectivas. In Sánchez C, Muñoz E, Alarcón E, eds. España siglo Xxı. Vol. 4: Ciencia y Tecnología. Madrid: Biblioteca Nueva; 2009. p. 9-32

6. Sánchez C, Muñoz E, Alarcón E, eds. España siglo xxi. Vol. 4: Ciencia y Tecnología. Madrid: Biblioteca Nueva; 2009.

7. Nieto-Galán A. Los públicos de la ciencia. Expertos y profanos a través de la historia. Madrid: Marcial Pons; 2011.

8. Nieto-Galán A. Ciencia popular. La historia de la ciencia nos ayuda a comprender mejor la comunicación entre expertos y profanos. Investigación y Ciencia 2012; 425: 46-7.

9. Silva LC. La investigación biomédica y sus laberintos. En defensa de la racionalidad para la ciencia del siglo XXI. Madrid: Díaz de Santos; 2009.

10. Cereijido M. La ciencia como calamidad. Un ensayo sobre el analfabetismo científico y sus efectos. Barcelona: Gedisa; 2012.

11. Olivé L. El bien, el mal y la razón. Facetas de la ciencia y de la tecnología. Barcelona: Paidós; 2000. 
12. Olivé L. La ciencia y la tecnología en la sociedad del conocimiento. México DF: Fondo de Cultura Económica; 2007.

13. Muñoz van den Eynde A. Conocimiento científico y conciencia ambiental. In López-Cerezo JA, Gómez-González FJ, eds. Apropiación social de la ciencia. Madrid: Biblioteca Nueva; 2008. p. 91-114.

14. Elena A, Ordóñez J. La ciencia y su público. Madrid: Consejo Superior de Investigaciones Científicas; 1990

15. Rivera A. La mitad de los españoles no es capaz de citar un solo científico. El País, 9 de mayo de 2012.

16. Ibarra A. La democratización de la ciencia. Donostia: EREIN 2003.

17. López-Cerezo JA, Gómez-González FJ. Apropiación social de la ciencia. Madrid: Biblioteca Nueva; 2008.

18. Ordóñez J. Ciencia, tecnología e historia. 2 ed. Madrid: Fondo de Cultura Económica de España; 2003.

19. Alcíbar M. Comunicar la ciencia. La clonación como debate periodístico. Madrid: CSIC; 2007.

20. Elías C. Fundamentos de periodismo científico y divulgación mediática. Madrid: Alianza Editorial; 2008.

21. Díaz-Rojo JA. Retórica científica en la prensa: el hallazgo paleontológico del cráneo de Orce (1983). In Díaz-Rojo JA, ed. La circulación del saber científico en los siglos xix y xx. Valencia: Universitat de València; 2011. p. 99-128.

22. Díaz-García I, Muñoz van den Eynde A. Participación y cultura científica en contexto internacional. Madrid: Ediciones de la Catarata/CSIC; 2011.

23. González-Alcaide G, Valderrama-Zurián JC, AleixandreBenavent R. La investigación sobre la divulgación de la ciencia en España: situación actual y retos para el futuro. ARBOR 2009; 185: 861-9.

24. Hidalgo A, Bordallo J, Sánchez M, Cantabrana B. Protagonismo de los alumnos en el aprendizaje. Una experiencia en el primer curso de medicina. Educ Med 2012; 15: 213-9.

25. Bunge M. Cápsulas. Madrid: Gedisa; 2003.
26. García-Blanco JM, Fazio ME. Percepciones, imaginario y apropiación social de la ciencia y la tecnología: comparaciones entre España, la Unión Europea y Estados Unidos. In LópezCerezo JA, Gómez-González FJ, eds. Apropiación social de la ciencia. Madrid: Biblioteca Nueva; 2008. p. 19-62.

27. Ordóñez-Gallego A. Lenguaje médico. Modismos, tópicos y curiosidades. Madrid: Noesis; 1994.

28. López-Piñero JM, Terrada-Ferrandis ML. Introducción a la terminología médica. 2 ed. Barcelona: Masson; 2005

29. Martínez-Fresneda $\mathrm{H}$. El protagonismo de los medios de comunicación en la formación del alumno. Comunicar 2006; 26: 143-8.

30. Calvo-Hernando M. El periodismo científico, reto de las sociedades del siglo XxI. Comunicar 2002; 19: 15-8.

31. Macián C. Saber y comunicar: la proyección social de la ciencia. Panace@ 2006; 7: 348-9.

32. Cámara-Hurtado M, Díaz-Martínez JA, López-Cerezo JA, Luján JL, Martín-Sempere MJ, Moreno-Castro C, et al. Percepción social de la ciencia y la tecnología en España 2008 [Internet]. Madrid: Fundación Española para la Ciencia y la Tecnología; 2009. URL: http://icono.fecyt.es/ informesypublicaciones/Documents/percepcin_social_ cyt_2008_web.pdf. [01.02.2013].

33. De Semir V. Meta-análisis: comunicación científica y periodismo científico. Madrid: Fundación Española para la Ciencia y la Tecnología; 2011.

34. Domínguez M. Divulgar la investigación con revistas científicas: el caso de Mètode. Comunicar 2002; 19: 49-53.

35. Membiela P, Padilla Y, eds. Retos y perspectivas de la enseñanza de las ciencias desde el enfoque ciencia/tecnología/sociedad en los inicios del siglo XxI. Vigo: Educación Editora; 2005.

36. Martín-Díaz V. Medios de comunicación, educación y realidad. Comunicar 2006; 26: 193-7.

37. Gabelas JA. Una perspectiva de la educación en medios para la comunicación en España. Comunicar 2007; 28: 69-73. 